The study evaluated outcomes for 134 children with medulloblastoma who sequentially received tumor resection, risk-adjusted craniospinal radiotherapy (23.4Gy for average-risk disease; 36.0-39.6Gy for high-risk disease), and 4 months of high-dose chemotherapy. Estimated 5-year event-free survival was 83\% for patients with average risk and $70 \%$ for those with high risk $(P=0.046)$. Tumor histological subtype also predicted event-free survival after adjustment for clinical stage, with large-cell anaplastic tumors conferring a poor survival rate (57\%) compared with classic histology (84\%) or desmoplastic tumor $(77 \% ; P=0.04)$.

In this protocol, both radiotherapy and chemotherapy were well tolerated, and there were no treatment-related deaths. The authors suggest that the high observed survival rates might be associated with the initiation of radiotherapy soon (within 4 weeks) after resection, and with the use of shortterm dose-intensive chemotherapy following completion of radiotherapy.

Original article Gajjar A et al. (2006) Risk-adapted craniospinal radiotherapy followed by high-dose chemotherapy and stemcell rescue in children with newly diagnosed medulloblastoma (St Jude Medulloblastoma-96): long-term results from a prospective, multicentre trial. Lancet Oncol 7: 813-820

\section{Early-stage Hodgkin's disease can be treated successfully with mantle irradiation alone}

In an Annals of Oncology paper, $\mathrm{Ng}$ et al. report the long-term results of a prospective trial assessing the efficacy of mantle radiation therapy alone in patients with early-stage, favorable prognosis Hodgkin's disease. Although combined modality treatment is now preferred for early-stage Hodgkin's disease, at the time of study initiation extended-field radiation was standard therapy; compared with this modality, mantle irradiation alone is associated with fewer side effects and a reduced risk of late effects such as second malignancies. During the period 1988-2000, 87 patients with pathologic stage IA-IIA or clinical stage IA Hodgkin's disease were enrolled in the singlearm trial. Each patient received a dose of radiation that was dependent upon the initial sites of involvement. The median doses of radiation to the mantle and mediastinum fields were 36 Gy and 38.6 Gy, respectively.
The 10-year actuarial freedom from treatment failure was $84.7 \%$ and the 10 -year overall survival rate was $98.2 \%$. In total, 13 patients relapsed at a median of 30 months following treatment, but after salvage therapy all 13 patients were still alive at publication. The 10-year actuarial risk of a second malignancy was $4.5 \%$. To date there have been two deaths, both caused by second malignancies.

The authors conclude that, while combined modality therapy should remain the treatment of choice in patients with early-stage Hodgkin's disease, mantle irradiation alone achieves a good overall survival rate, and might prove useful in patients who would benefit from being spared the toxicity associated with chemotherapy.

Original article $\mathrm{Ng} \mathrm{AK}$ et al. (2006) Long-term results of a prospective trial of mantle irradiation alone for early-stage Hodgkin's disease. Ann Oncol 17: 1693-1697

\section{Melanoma micrometastases $(<0.1 \mathrm{~mm})$ in sentinel nodes can be regarded as negative}

Around $20 \%$ of melanoma patients with a positive sentinel node (SN) also have nonSN lymph-node involvement and require complete lymph-node dissection (CLND). Currently, most SN-positive patients with melanoma undergo CLND; the procedure is unnecessary in many of these cases. van Akkooi et al. have evaluated factors that might identify a subgroup of SN-positive patients who can be spared CLND, and its associated morbidity, without reducing their chances of survival.

This study comprised 74 SN-positive patients aged 16-76 years, followed for a mean of 35 months. Multivariate analysis revealed SN tumor burden to be an independent predictor of disease-free survival $(P=0.005)$ and overall survival $(P=0.03)$ at 5 years. Estimated 5 -year overall survival rates were $100 \%$ for patients with micrometastases ( $>10$ cells, $<0.1 \mathrm{~mm}$ ), $63 \%$ for patients with tumors $0.1-1.0 \mathrm{~mm}$, and $35 \%$ for patients with tumors $>1.0 \mathrm{~mm}$. For patients with micrometastases, the study found no additional non-SN lymph-node involvement, and the 5-year distant-metastasis-free survival rate of $91 \%$ was comparable with the overall survival rate of $\mathrm{SN}$-negative patients.

The authors conclude that patients with SN micrometastases ( $22 \%$ of patients in this study) 